\title{
Genome-edited crops: how to move them from laboratory to market
}

\author{
Kunling CHEN ${ }^{1}$, Caixia GAO (ه) $)^{1,2}$ \\ 1 State Key Laboratory of Plant Cell and Chromosome Engineering, Center for Genome Editing, Institute of Genetics and Developmental \\ Biology, Innovation Academy for Seed Design, Chinese Academy of Sciences, Beijing 100101, China \\ 2 College of Advanced Agricultural Sciences, University of the Chinese Academy of Sciences, Beijing 100101, China
}

\begin{abstract}
Recent breakthroughs in CRISPR technology allow specific genome manipulation of almost all crops and have initiated a revolution in precision crop breeding. Rationally-based regulation and widespread public acceptance are needed to propel genome-edited crops from laboratory to market and to translate this innovative technology into agricultural productivity.
\end{abstract}

Keywords CRISPR/Cas, genome editing, base editing, precision breeding, regulation

\section{Introduction}

The main task of modern agriculture is to feed the rapidly growing human population under conditions of reduced arable land area and water resources and a changing climate. Agriculture has increasingly benefited from elite crop cultivars produced by advanced breeding technology since the first Green Revolution increased wheat and rice yields by exploiting semi-dwarf genes in the 1960s. Conventional breeding methods (cross breeding and mutation breeding) as well as transgenic breeding have made enormous contributions to increased food production, but new cost-effective and safe approaches are needed to meet future demand. Currently, cross breeding improves cultivars by crossing compatible germplasm to introduce genetic variants and by selecting outstanding progeny with the desired trait ${ }^{[1]}$. Mutation is induced by exposure to mutagens such as ethyl methanesulfonate (which mainly induces single base $\mathrm{C}$ to $\mathrm{T}$ and $\mathrm{G}$ to $\mathrm{A}$ substitutions) and irradiation with, for example, X-rays (which usually induce small deletions of no more than $20 \mathrm{bp})^{[2]}$. All these conventionally bred cultivars have good safety records and fall outside regulatory controls worldwide.

Received December 9, 2019; accepted January 22, 2020

Correspondence: cxgao@genetics.ac.cn
Transgenesis produces genetically-modified (GM) crops by transferring foreign genes into elite background cultivars. However, the procedure raises concern in terms of potential risks to health and the environment due to the random integration of foreign DNA into the host genome. This has provoked strict regulation and permission is required for commercial exploitation in most countries ${ }^{[3]}$.

Genome editing, involving targeted manipulation of the genome by site-directed nucleases (SDNs), provides a powerful tool for precision crop breeding. The great advantage of this technology is that it can introduce novel traits without threatening the stability of the whole genome, and the genome-edited plants cannot be distinguished from unmodified plants. The clustered regulatory interspaced short palindromic repeats (CRISPR)/CRISPRassociated protein (Cas) systems are the most widely adopted SDN ${ }^{[4]}$. Precision plant gene editing was selected as one of the ten breakthrough technologies in 2016 by the MIT Technology Review because of the contribution of its great potential to solve the future food crisis. The prospect of a new green revolution made possible by genome editing is approaching. However, most research has been in the form of proof of concept. Scientifically-based regulation of genome edited crops (GECs), positive and effective public communication, and optimization and development of the technology are key elements needed to propel these novel products from the laboratory to the marketplace worldwide to benefit from this innovation.

\section{Development and application of CRISPR genome editing technologies for precision crop breeding}

Plant CRISPR genome editing has made great progress ${ }^{[4]}$ since the first use of CRISPR/Cas9 in plants was reported in $2013^{[5-7]}$. Cas9, Cas12a, Cas12b, Cas13a, Cas13b and many variants demonstrated DNA or RNA editing abilities have been added to the toolbox. Targeted gene knockout, 
gene replacement, gene insertion, and multiplex editing with CRISPR/Cas9 or CRISPR/Cas12a have been conducted in many plant species.

CRISPR reagents (Cas/sgRNA) are delivered into plant cells via Agrobacterium-mediated transformation or particle bombardment to generate edited crops, and mutant plants are regenerated from the edited cells by tissue culture $^{[8]}$. Normally, the Cas/sgRNAs induce DNA double strand breaks (DSBs) which recruit the DNA repair pathways of non-homologous end joining (NHEJ) and homologous repair (HR), leading to editing via gene knockout, replacement or insertion (Fig. 1). The NHEJ repair pathway, which is supplied with an SDN only, is an error-prone repair pathway and usually induces insertions of a small number of base pairs or small deletions (indels) at the breaks. Given that NHEJ is the predominant repair pathway, targeted sequence mutations (which we classify as SDN-1) are the most common outcome. The HR repair pathway, supplied with homologous DNA templates along with SDNs, is used to introduce specific point mutations or sequence replacements (SDN-2) or to insert desired sequences (SDN-3) at the target site. Also, the programmable DNA binding ability of the CRISPR/Cas system provides a versatile platform for recruitment of different functional domains to target sites. Precise base pair substitutions can also be generated even in the absence of DSBs.

Base editing (BE) provides an efficient and simple strategy for obtaining base pair substitutions independent of HR and without generating DSBs and providing template DNA. The main principle of $\mathrm{BE}$ is to fuse a deaminase to nCas9 or dCas $9^{[9]}$. The sgRNA directs the nCas9/dCas9-deaminase to the target sequence and the deaminase converts the targeted base pairs in the editing window. Two types of base editors, cytosine base editors and adenine base editors, have been used in plants. Most recently, Anzalone et al. have introduced prime editing $(\mathrm{PE})$ in which an engineered reverse transcriptase and a PE guide RNA are combined with CRISPR/nCas ${ }^{[10]}$. This technology involves no DSBs or donor DNA template and is a powerful and versatile search-and-replace tool that can generate all 12 base-to-base conversions as well as targeted deletions and insertions, and even combinations of these changes. There is no doubt that PE will soon be applied to crop plants.

In addition, the development of genome editing methods that do not involve the use of foreign DNA is likely to overcome regulatory barriers and alleviate public concern. Using purified Cas9 and in vitro-transcribed sgRNAs to form ribonucleoproteins (RNPs), DNA-free genome editing methods have been used in Arabidopsis, lettuce, maize, rice, tobacco and wheat ${ }^{[11-13]}$.

\section{Applications in precision crop breeding: from trait improvement to new crops}

Substantial progress has been made in the field of precision crop breeding with the development of CRISPR technologies. Applications have varied from trait improvement to innovative cross breeding and exploited in creating new crops by de novo domestication of wild species ${ }^{[7]}$. The main contributions of CRISPR have been improvements in key agronomic traits and the incorporation of novel traits that could not be obtained by cross breeding methods.

\subsection{SDN-1 mediated trait improvement}

SDN-1 has been used to create loss-of-function null mutants by generating a frameshift or introducing a stop

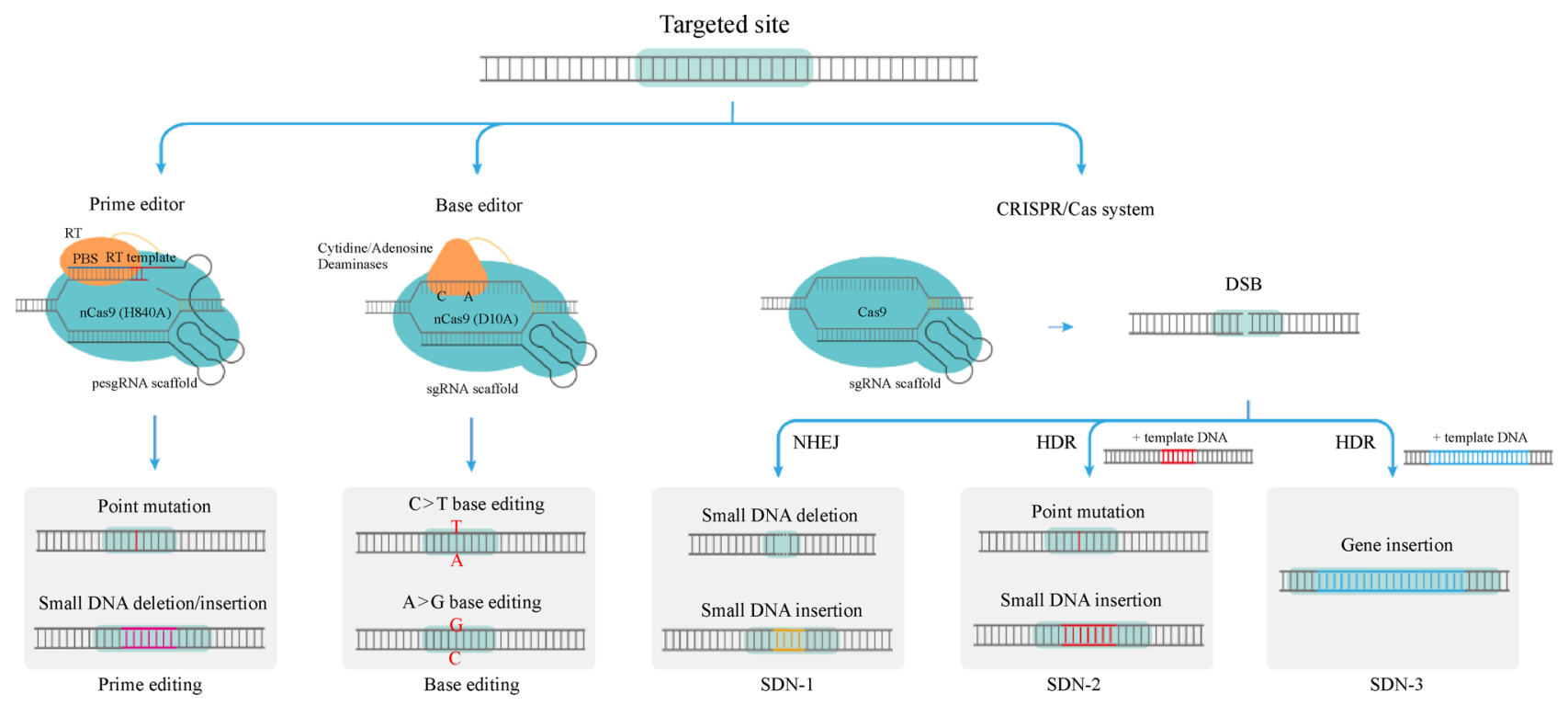

Fig. 1 Classification of CRISPR/Cas genome editing techniques according to DNA repair mechanism. 
codon. The most common SDN-1-mediated trait improvements have involved knocking out negative control genes or elements in order to improve yield or quality, or increase biotic- or abiotic-stress resistance. For instance, powdery mildew is a devastating worldwide disease of cereal crops. No natural hexaploid bread wheat germplasm with durable resistance to powdery mildew has been discovered due to the genetic redundancy of bread wheat. In 2014, Wang et al. generated powdery mildew-resistant wheat ${ }^{[14]}$ using TALEN and CRISPR to disrupt all three homeologs of the TaMLO gene, and in so doing, genome editing created genetic variants that cross breeding could not. The capacity of CRISPR to perform multiplex editing has allowed the development of the procedure of trait stacking by which $\mathrm{Xu}$ et al. simultaneously knocked out three grain weightrelated genes ( $G W 2, G W 5$ and $T G W 6)$ to increase rice grain weight ${ }^{[15]}$. More importantly, Sanchez-Leon et al. created a low-gluten wheat designed for patients with celiac disease ${ }^{[16]}$ through simultaneous knockout of most of the conserved domains of nearly $100 \alpha$-gliadin family members. In addition to disrupting gene function by deleting coding sequences, it has proved possible to finetune gene regulation by knocking out regulatory elements. Zhang et al. showed that disrupting endogenous upstream open reading frames (uORFs) could increase the translation of certain mRNAs ${ }^{[17]}$. Thus, by editing the uORF of $L s G G P 2$, they obtained a modified lettuce with about $150 \%$ increased ascorbate content. Recently, targeted disruption of the promoter of rice SWEET gene was shown to be an efficient solution to rice bacterial blight ${ }^{[18]}$.

Concurrently, genome editing has removed some crossbreeding bottlenecks. For example, hybridization is a very successful approach for improving crop productivity and adaptability, but the heterosis of F1 hybrids is not retained in subsequent generations and the cost of hybrid seed production is prohibitive. Through substituting mitosis for meiosis by simultaneously knocking out three key meiotic genes, REC8, PAIR1 and OSD1, Khanday et al. ${ }^{[19]}$ and Wang et al. ${ }^{[20]}$ independently were able to fix hybrid vigor by propagating seeds produced either by specific expression of $B B M 1$ in the egg or knocking out $M T L$ in hybrid rice.

\subsection{SDN-2 and SDN-3 mediated trait improvements}

SDN-2-mediated gene replacement and SDN-3-mediated gene insertion can confer traits by producing base pair substitutions or gene expression changes or by adding new function; hence, they are of great value in crop improvement. Trait improvements through gene replacement or gene insertion have been limited so far due to the low efficiency of the HR pathway. The most impressive example to date is the production of drought tolerant maize. Shi et al. increased ARGOS 8 expression by substituting a stronger GOS2 promoter in place of the native promoter ${ }^{[21]}$. Herbicide resistance traits, which can be enriched by selection, are also a good example of this. For example, Sun et al. created sulfonylurea-based herbicide-resistant rice through HR-generated base substitutions in $A L S^{[22]}$. TIPS EPSPS glyphosate-resistant rice was created based on an intron-targeting strategy using NHEJ instead of the less efficient HR-mediated gene replacement and insertion ${ }^{[23]}$.

\subsection{Trait improvements developed by BE}

Single nucleotide polymorphisms (SNPs) are the main source of genetic diversity. Introducing elite SNPs into modern cultivars is therefore an efficient approach to crop improvement. A popular application of BE has been to introduce base pair changes in target genes that confer herbicide resistance in crops such as cotton, maize, rice, watermelon and wheat. Wheat strains with tolerance to multiple herbicides such as sulfonylurea-, imidazolinoneand aryloxyphenoxy propionate-type herbicides have recently been generated by $\mathrm{BE}^{[24]}$.

\subsection{Creating new crops}

Plant domestication can be traced back to over 10000 years ago. Direct selection applied to wild plants over a long time has succeeded in increasing productivity but has often been accompanied by loss of fitness and of genetic diversity. $D e$ novo domestication of wild species to create new crops by genome editing has been achieved ${ }^{[25-27]}$. For example, by multiplex editing of coding regions of SELF-PRUNING and $S E L F-P R U N I N G 5 G$, together with cis-regulatory regions of $C L V 3$ and $W U S$ or uORFs of GGP1, Li et al. generated a de novo domesticated tomato with compact plant architecture, synchronized fruit ripening, day length insensitivity, enlarged fruit size and increased vitamin C levels ${ }^{[25]}$. These innovations represent successful attempts at developing new improved crops in order to avoid future crises and maintain food security in sustainable low-input agricultural economies.

\section{The global regulatory landscape for genome-edited crops}

Since last century biotechnology regulations have been ratified in most countries to ensure that crops that have been genetically modified by modern biotechnology are as safe as conventional products of traditional breeding ${ }^{[28]}$. Product-based and process-based regulation are alternative regulatory frameworks adopted in different countries and regions ${ }^{[29]}$. In product-based regulation, only the improved trait and the biological product itself are the objects of regulation; conversely, process-based regulation is based the use of a GM process.

GECs do not fit well in the existing GMO regulatory framework as a result of the explosion in technological 
innovations, and it seems time to refine the current regulatory rules to fit the new products. More and more countries such as Argentina, Australia, Brazil, Canada, Chile, Japan and the United States are starting to amend their rules to deregulate certain GECs. In 2015, using a case-by-case process, product-based Argentina created the world's first legislation (Resolution 173, 2015) that accepted that new plant breeding technology bred plants (including GECs), without novel recombination and having no resident transgenes, need not be covered by GMO regulations ${ }^{[30]}$. Other South American countries including Chile, Brazil and Colombia have announced similar decisions ${ }^{[31]}$. Regulation in Canada, a typical country with product-based regulation, depends on the novelty of the created trait: if it is not novel, then it does not need to be regulated regardless of what breeding method is adopted $^{[32]}$. Despite adopting a process-based regulatory framework, Australia opened the door to the planting and commercialization of GECs in 2019. It announced that if no new genetic material was introduced, genetic edits made without templates (such as SDN-1) were exempt from regulation ${ }^{[33]}$. Also, GECs modified by DNA-free editing with Cas9/sgRNA RNP were not subject to regulation since from the step of modification occurs. In the same year, Japan announced a new policy according to which loss-of-function GECs without resident foreign DNA were the same as non-GECs and GE foods and were permitted in the market while registration was pending ${ }^{[34]}$. The United States prefers a case-by-case product-based regulatory approach. According to a USDA statement in 2018, GECs based on deletions or single base pair substitution will not be regulated as long as they are not plant pests or have been developed using plant pests ${ }^{[35]}$. Many GECs such as powdery mildew resistant wheat, drought and salt tolerant soybean, camelina with increased oil content, waxy corn and anti-browning mushrooms have already received approval for commercial use. In March 2019, Calyxt announced that Calyno ${ }^{\mathrm{TM}}$ high oleic soybean oil produced by GE soybeans would shortly be on the market in the USA. In 2018, 13 nations including Argentina, Australia, Brazil, Canada and the USA issued a joint statement which agreed to support agricultural applications of precision biotechnology ${ }^{[36]}$.

In contrast, under a process-based regulatory framework, the EU following European GMO Directive 2001/ 18/EC declared in 2018 that all the above GECs were subject to GMO regulation ${ }^{[37]}$. Many other countries including China are watching developments and have not yet decided how to regulate GECs. Worldwide delays in legislation have increased the time-lag between the rapid development of GECs and market commercialization.

\section{Scientific aspects of regulation of genetically-edited crops}

In view of the major benefits to human life expected from GE innovations, Huang et al. and their colleagues from the USA, China and Germany have proposed a five-step product-focused regulatory framework for GECs ${ }^{[38]}$. This proposal provides scientific guidelines for the development of regulations. To provide a rational basis for regulation, we suggest classifying GECs according to the nature of the products and the editing method used.

As described before, there are three types of DSBdependent editing methods (Fig. 2). SDN-1, small indel mutations without template DNA, have something in common with changes produced by random mutagenesis

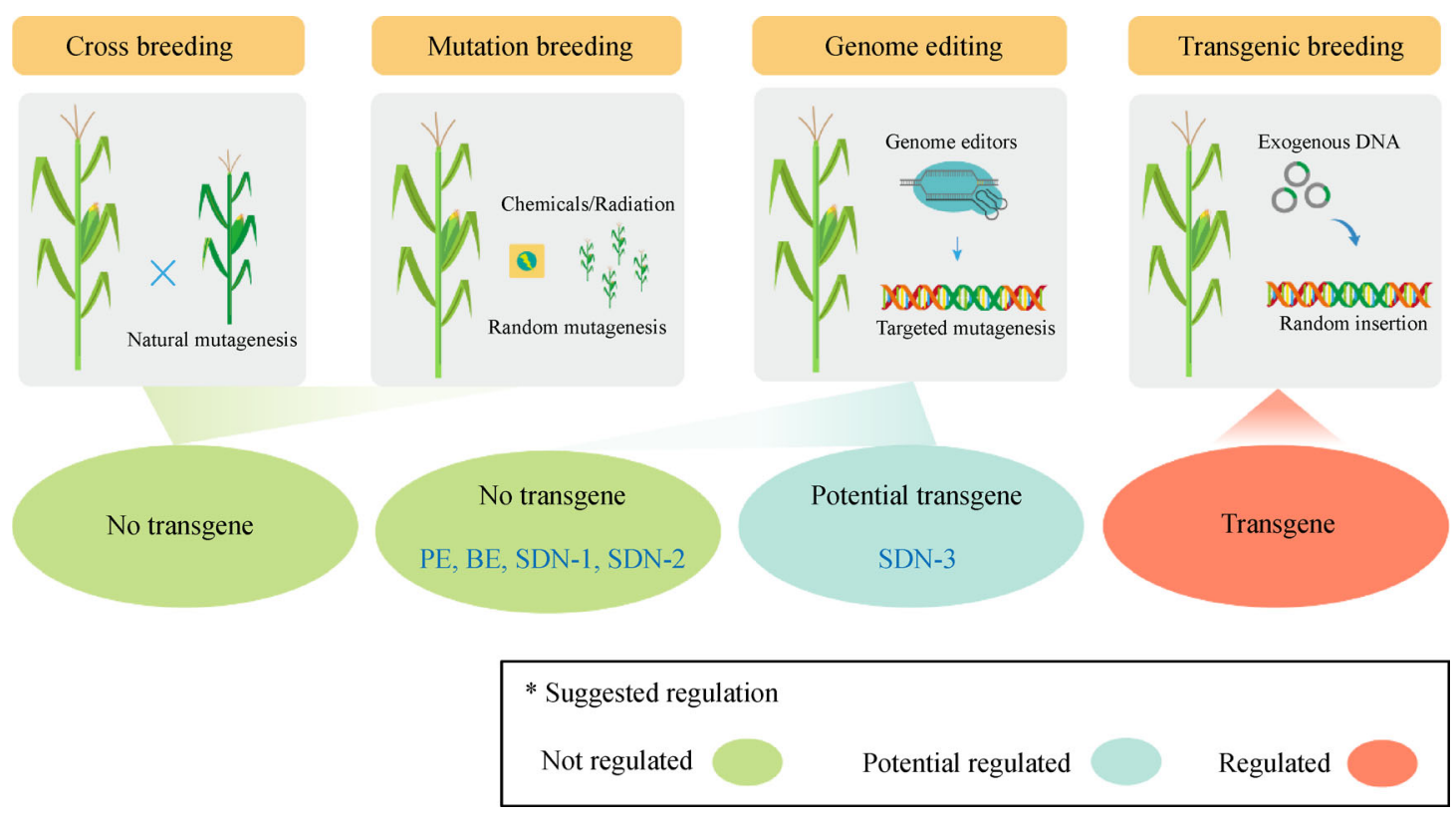

Fig. 2 Scientific regulation of GECs developed by different genome editing techniques. 
but are much more specific and straightforward. SDN-2, point mutations or sequence replacements made in conjunction with short template DNAs but identical to their target sequences except for a few base pairs and resemble normal DNA repair involving different alleles. SDN-3, with inserts of new DNA sequences at predetermined sites, are more specific than random insertions and avoid the side-effects of the latter. There are also two other types of editing methods (BE and PE) that are the result of DSB-independent editing. BE, single base pair substitutions without the involvement of DSBs or template DNA, are essentially alternatives to SDN-2. PE provides a complementary method for generating genetic changes, such as precise deletions and nucleotide substitutions, that cannot be achieved with current genome editing tools in plants.

The GECs produced by SDN-1, BE and PE do not contain any exogenous DNA fragments, but carry substitutions or other DNA modifications similar to or indistinguishable from mutants obtained by mutagenesis. So, from a scientific and safety perspective, if no novel traits are introduced, these products should be treated like conventionally bred cultivars exempted from GMO regulation (Fig. 2). In the case of GECs produced by SDN-2, regulation should depend on the length of the template DNA used; provided it is shorter than $20 \mathrm{bp}$, regulation should not be needed. Finally, GECs produced by SDN-3 should be subject to GMO regulation, either process- or product-based.

\section{Challenges and prospects}

Genome editing is an accurate and timesaving innovation that provides an unprecedented ability to facilitate precision crop breeding. However, most of the achievements, genetically-edited crop plants, remain as experimental products in laboratories. The challenges for both science and society need to be carefully considered.

In terms of the science, target gene selection and genome editing are the two key steps in producing a precision crop breeding pipeline. The discovery of target genes related to important agronomic traits and further developments in genome editing are therefore the two most important tasks. Although low cost genome sequencing technology has yielded more than 200 plant genome sequences ${ }^{[39]}$, there are only a few candidate genes for editing. A bottleneck to discovering target genes is in linking the very large amount of available sequence data to specific traits. This will require developing large scale approaches to connecting phenotype with genotype to efficiently detect trait-gene associations, and to identify and prioritize candidate genes that promote crop improvement. Another challenge of CRISPR technology is the low efficiencies of delivery systems, which greatly impedes the widespread adoption of genome editing of crops. The major delivery systems currently established in crops are inefficient, tissue culturedependent and restricted to a limited number of genotypes in a few crop species. Increasing delivery efficiency and developing genotype-independent and tissue culture-free delivery systems via the germ line or meristematic cells are therefore key challenges.

Governments, developers, growers, and consumers all play important roles in the genome editing economy chain. Active cooperation and communication between them are critical to enhance GECs and related products and move them into the market. Governments should protect the benefits of all parts, thus rational regulatory mechanisms are required to manage GEC production and guarantee that all information is open to the public. Public trust in developers impacts the perception of benefits and the risk of GECs too. Traceable systems for GEC products will be helpful. At this stage, independent licensed organizations may help to narrow the gap between developers and consumers and to evaluate scientifically the safety of GECs and products. Consumers will be the most important factor in commercializing genome editing products. However, persuading people of different educational backgrounds to trust novel agricultural products, given the widespread conservatism about GM foods and products, is a major challenge. Hence, positive and effective public communication to help the public understand this novel technology, and at least to treat GE food differently from GM food, would be a start. In this situation we strongly suggest creating a regulatory framework based not only on scientific considerations but also on public acceptance. We recommend that researchers use DNA-free editing with Cas9 RNP and base editor RNP to produce GECs and that the regulatory authorities be more open with the public since transparency should help gain public trust.

Genome editing has been used as a new plant breeding technique and genome-edited crops are on the point of commercialization. However, whether genome editing will revolutionize agriculture and realize its tremendous potential for crop breeding will depend on how regulators and society respond.

Acknowledgements We thank Yanpeng Wang and Qiupeng Lin (Institute
of Genetics and Developmental Biology, Chinese Academy of Sciences) for
help with the figures. This work was supported by grants from the National
Natural Science Foundation of China ( 31788103 and 31971370) and the
National Transgenic Science and Technology Program (2016ZX08010002
and 2018ZX0801002B).

Compliance with ethics guidelines Kunling Chen and Caixia Gao declare that they have no conflicts of interest or financial conflicts to disclose.

This article is a review and does not contain any studies with human or animal subjects performed by any of the authors.

\section{References}

1. Scheben A, Wolter F, Batley J, Puchta H, Edwards D. Towards CRISPR/Cas crops-bringing together genomics and genome 
editing. New Phytologist, 2017, 216(3): 682-698

2. Pacher M, Puchta H. From classical mutagenesis to nuclease-based breeding - directing natural DNA repair for a natural end-product. Plant Journal, 2017, 90(4): 819-833

3. Prado J R, Segers G, Voelker T, Carson D, Dobert R, Phillips J, Cook K, Cornejo C, Monken J, Grapes L, Reynolds T, Martino-Catt S. Genetically engineered crops: from idea to product. Annual Review of Plant Biology, 2014, 65(1): 769-790

4. Chen K, Wang Y, Zhang R, Zhang H, Gao C. CRISPR/Cas genome editing and precision plant breeding in agriculture. Annual Review of Plant Biology, 2019, 70(1): 667-697

5. Li J F, Norville J E, Aach J, McCormack M, Zhang D, Bush J, Church G M, Sheen J. Multiplex and homologous recombinationmediated genome editing in Arabidopsis and Nicotiana benthamiana using guide RNA and Cas9. Nature Biotechnology, 2013, 31(8): 688-691

6. Nekrasov V, Staskawicz B, Weigel D, Jones J D, Kamoun S. Targeted mutagenesis in the model plant Nicotiana benthamiana using Cas9 RNA-guided endonuclease. Nature Biotechnology, 2013, 31(8): 691-693

7. Shan Q, Wang Y, Li J, Zhang Y, Chen K, Liang Z, Zhang K, Liu J, Xi J J, Qiu J L, Gao C. Targeted genome modification of crop plants using a CRISPR-Cas system. Nature Biotechnology, 2013, 31(8): 686-688

8. Yin K, Gao C, Qiu J L. Progress and prospects in plant genome editing. Nature Plants, 2017, 3(8): 17107

9. Mishra R, Joshi R K, Zhao K. Base editing in crops: current advances, limitations and future implications. Plant Biotechnology Journal, 2020, 18(1): 20-31

10. Anzalone A V, Randolph P B, Davis J R, Sousa A A, Koblan L W, Levy J M, Chen P J, Wilson C, Newby G A, Raguram A, Liu D R. Search-and-replace genome editing without double-strand breaks or donor DNA. Nature, 2019, 576(7785): 149-157

11. Woo J W, Kim J, Kwon S I, Corvalán C, Cho S W, Kim H, Kim S G, $\operatorname{Kim} \mathrm{S}$ T, Choe S, Kim J S. DNA-free genome editing in plants with preassembled CRISPR-Cas9 ribonucleoproteins. Nature Biotechnology, 2015, 33(11): 1162-1164

12. Svitashev S, Schwartz C, Lenderts B, Young J K, Mark Cigan A. Genome editing in maize directed by CRISPR-Cas9 ribonucleoprotein complexes. Nature Communications, 2016, 7(1): 13274

13. Liang Z, Chen K, Li T, Zhang Y, Wang Y, Zhao Q, Liu J, Zhang H, Liu C, Ran Y, Gao C. Efficient DNA-free genome editing of bread wheat using CRISPR/Cas9 ribonucleoprotein complexes. Nature Communications, 2017, 8(1): 14261

14. Wang Y, Cheng X, Shan Q, Zhang Y, Liu J, Gao C, Qiu J L. Simultaneous editing of three homoeoalleles in hexaploid bread wheat confers heritable resistance to powdery mildew. Nature Biotechnology, 2014, 32(9): 947-951

15. Xu R, Yang Y, Qin R, Li H, Qiu C, Li L, Wei P, Yang J. Rapid improvement of grain weight via highly efficient CRISPR/Cas9mediated multiplex genome editing in rice. Journal of Genetics and Genomics, 2016, 43(8): 529-532

16. Sánchez-León S, Gil-Humanes J, Ozuna C V, Giménez M J, Sousa C, Voytas D F, Barro F. Low-gluten, nontransgenic wheat engineered with CRISPR/Cas9. Plant Biotechnology Journal, 2018, 16(4): 902-910
17. Zhang H, Si X, Ji X, Fan R, Liu J, Chen K, Wang D, Gao C. Genome editing of upstream open reading frames enables translational control in plants. Nature Biotechnology, 2018, 36(9): 894-898

18. Oliva R, Ji C, Atienza-Grande G, Huguet-Tapia J C, Perez-Quintero A, Li T, Eom J S, Li C, Nguyen H, Liu B, Auguy F, Sciallano C, Luu V T, Dossa G S, Cunnac S, Schmidt S M, Slamet-Loedin I H, Vera Cruz C, Szurek B, Frommer W B, White F F, Yang B. Broadspectrum resistance to bacterial blight in rice using genome editing. Nature Biotechnology, 2019, 37(11): 1344-1350

19. Khanday I, Skinner D, Yang B, Mercier R, Sundaresan V. A maleexpressed rice embryogenic trigger redirected for asexual propagation through seeds. Nature, 2019, 565(7737): 91-95

20. Wang C, Liu Q, Shen Y, Hua Y, Wang J, Lin J, Wu M, Sun T, Cheng Z, Mercier R, Wang K. Clonal seeds from hybrid rice by simultaneous genome engineering of meiosis and fertilization genes. Nature Biotechnology, 2019, 37(3): 283-286

21. Shi J, Gao H, Wang H, Lafitte H R, Archibald R L, Yang M, Hakimi S M, Mo H, Habben J E. ARGOS8 variants generated by CRISPRCas9 improve maize grain yield under field drought stress conditions. Plant Biotechnology Journal, 2017, 15(2): 207-216

22. Sun Y, Zhang X, Wu C, He Y, Ma Y, Hou H, Guo X, Du W, Zhao $\mathrm{Y}$, Xia L. Engineering herbicide-resistant rice plants through CRISPR/Cas9-mediated homologous recombination of acetolactate synthase. Molecular Plant, 2016, 9(4): 628-631

23. Li J, Meng X, Zong Y, Chen K, Zhang H, Liu J, Li J, Gao C. Gene replacements and insertions in rice by intron targeting using CRISPR-Cas9. Nature Plants, 2016, 2(10): 16139

24. Zhang R, Liu J, Chai Z, Chen S, Bai Y, Zong Y, Chen K, Li J, Jiang $\mathrm{L}$, Gao C. Generation of herbicide tolerance traits and a new selectable marker in wheat using base editing. Nature Plants, 2019, 5(5): 480-485

25. Li T, Yang X, Yu Y, Si X, Zhai X, Zhang H, Dong W, Gao C, Xu C. Domestication of wild tomato is accelerated by genome editing. Nature Biotechnology, 2018, 36(12): 1160-1163

26. Lemmon Z H, Reem N T, Dalrymple J, Soyk S, Swartwood K E, Rodriguez-Leal D, Van Eck J, Lippman Z B. Rapid improvement of domestication traits in an orphan crop by genome editing. Nature Plants, 2018, 4(10): 766-770

27. Zsögön A, Čermák T, Naves E R, Notini M M, Edel K H, Weinl S, Freschi L, Voytas D F, Kudla J, Peres L E P. De novo domestication of wild tomato using genome editing. Nature Biotechnology, 2018, 36(12): 1211-1216

28. Ishii T. Crop gene-editing: should we bypass or apply existing GMO policy? Trends in Plant Science, 2018, 23(11): 947-950

29. Ishii T, Araki M. A future scenario of the global regulatory landscape regarding genome-edited crops. GM Crops and Food: Biotechnology in Agriculture and the Food Chain, 2017, 8(1): 4456

30. Whelan A I, Lema M A. Regulatory framework for gene editing and other new breeding techniques (NBTs) in Argentina. GM Crops and Food: Biotechnology in Agriculture and the Food Chain, 2015, 6 (4): 253-265

31. Zannoni L. Evolving regulatory landscape for genome-edited plants. CRISPR Journal, 2019, 2(1): 3-8

32. Smyth S J. Canadian regulatory perspectives on genome engineered crops. GM Crops and Food: Biotechnology in Agriculture and the 
Food Chain, 2017, 8(1): 35-43

33. Office of the Gene Technology Regulator (OGTR) of Australian Government of Department of Health. Overview of the Gene Technology Amendment (2019 Measures No. 1) Regulations 2001. Available at OGTR website on September 1, 2019

34. Li G, Liu Y G, Chen Y. Genome-editing technologies: the gap between application and policy. Science China Life Sciences, 2019, 62(11): 1534-1538

35. United States Department of Agriculture (USDA). Secretary Perdue Issues USDA Statement on Plant Breeding Innovation. Available at USDA website on March 28, 2018
36. Cameron J. 13 nations say it's time to end 'political posturing' and embrace crop gene editing. Available at Genetic Literacy Project website on November 7, 2018

37. Bruetschy $\mathrm{C}$. The EU regulatory framework on genetically modified organisms (GMOs). Transgenic Research, 2019, 28(Suppl 2): 169174

38. Huang S, Weigel D, Beachy R N, Li J. A proposed regulatory framework for genome-edited crops. Nature Genetics, 2016, 48(2): 109-111

39. Scheben A, Edwards D. Bottlenecks for genome-edited crops on the road from lab to farm. Genome Biology, 2018, 19(1): 178 